\title{
Téoros
}

Revue de recherche en tourisme

\section{La promotion du tourisme internationaldans le Pacifique}

\section{Pascal Tremblay}

Volume 6, numéro 3, décembre 1987

Le tourisme, phénomène mondial

URI : https://id.erudit.org/iderudit/1080602ar

DOI : https://doi.org/10.7202/1080602ar

Aller au sommaire du numéro

Éditeur(s)

Université du Québec à Montréal

ISSN

0712-8657 (imprimé)

1923-2705 (numérique)

Découvrir la revue

Citer cet article

Tremblay, P. (1987). La promotion du tourisme internationaldans le Pacifique. Téoros, 6(3), 19-21. https://doi.org/10.7202/1080602ar d'utilisation que vous pouvez consulter en ligne.

https://apropos.erudit.org/fr/usagers/politique-dutilisation/ 


\section{La promotion du tourisme international dans le Pacifique}

La région du "Pacific Rim" est née d'une division géographique et stratégique plus ou moins arbitraire et réunit les pays d'Asie de l'Est et d'Océanie baignant le Pacifique. Ils sont souvent regroupés à des fins pratiques mais rarement dans la réalité politique et économique. Ils ont cependant plusieurs points en commun.

La vaste région du Sud-Est Asiatique et du Pacifique Sud a pour principale caractéristique son éloignement des principaux pays émetteurs de touristes, provenant d'Europe et d'Amérique du Nord. Cette région a connu au cours des quinze dernières années un taux de croissance des arrivées de touristes très supérieur à la moyenne internationale. Par exemple, les arrivées de touristes internationaux se sont aocrues, en moyenne de $9.3 \%$ par année (contre $3.8 \%$ dans le monde) au cours de la période $1975-1982^{(1)}$. Cette forte croissance a êté accompagnée de changements dans la composition des lieux d'origine des touristes. Le changement majeur a été la croissance du tourisme intra-régional, principalement en provenance du Japon ${ }^{(2)}$. Ceci est dû à la croissance des échanges commerciaux intra-régionaux et du tourisme d'affaires qui en découle. Aussi, la région a vu naître des infrastructures touristiques de grande qualité et souvent couteuses.

Pour tenir compte des disparités économiques, culturelles et politiques du Pacifique, il faut décomposer ce territoire en quatre sous-régions; chacune se caractérise par une situation particulière de développement et conséquemment, par une stratégie propre de promotion.

\section{Les îles du Pacifique}

À l'instar des Caraïbes, les íles du Pacifique ont toujours su projeter une image associant les paysages de rêve à l'hospitalité des indigènes et à leurs cultures très diversifiées. Certaines de ces îles sont devenues rapidement des destinations de vacances privilégiées pour les australiens et les néo-zélandais (et récemment pour les japonais) et sont littéralement inondées de touristes à certaines saisons. Ce tourisme "intensif" a marqué brutalement ces économies peu diversifiées et a exigé des ajustements rapides de la part des gouvernements qui y ont vu surtout des

-Pascal Tremblay, diplómé en gestion et intervention touristiques à l'UOAM, a fait ses études de maitrise en sciences économiques en Colombie-Britannique et est actuellement professeur au Département d'économique de I'Université de Tasmanie, à Haubart en Australie.

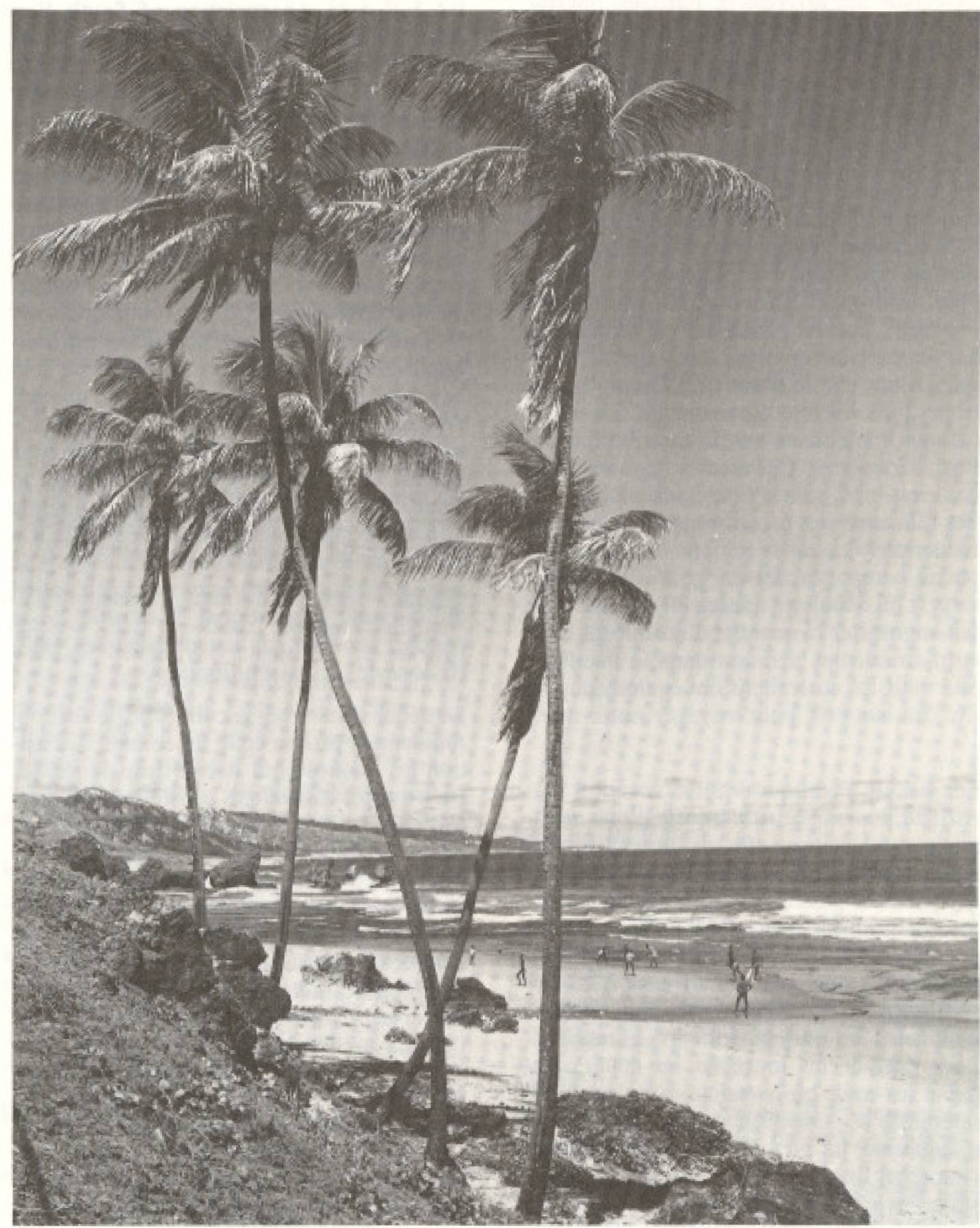

gains économiques alléchants. La situation actuelle, pour les cas les mieux connus (Fiji, Tonga, Samoa oriental, Iles Cook, NouvelleCalédonie), se traduit par une dépendance économique au moins partielle envers le tourisme et par la détérioration du capital touristique. Tant pour la détérioration du capital humain (la gentillesse des indigènes) que pour le décor ou les infrastructures, la situation reste précaire car les îles ont besoin d'une croissance soutenue pour justifier leurs investissements et accroitre les revenus de leurs gouvernements.

Le cas de Fiji est bien connu et les avantages et désavantages du tourisme pour un petit pays en voie de développement ont déjà fait couler beaucoup d'encre ${ }^{(3)}$. Le problème particulier des fuites de devises touristiques (dues à la proportion du produit touristique qui est importée) a un impact majeur sur les bénéfices que ces pays tirent du tourisme et sur le choix des marchés internationaux. Par exemple, la croissance élevée (en proportion) du tourisme en provenance du Japon affec- 
tera le choix des activités d'animation de ces touristes. Les japonais, en effet, sont des touristes très mobiles qui veulent être constamment occupés. Si leur part du marché continue a croiltre, certaines infrastructures pourraient s'avérer rapidement inadéquates.

L'attitude des gouvernements a ếté jusqu'à présent de considéter le tourisme international comme souhaitable ou du moins, inévitable et d'essayer d'obtenir une croissance maximale des arrivées de touristes internationaux. Une des principales strategies adoptées fut d'assurer des liaisons aériennes aussi fréquentes et diversifiées que possibles. Ces petits pays ont dû faire des concessions aux grossistes et aux hôtels qui sont lies à des compagnies aériennes afin de s'assurer l'intérêt des transporteurs importants. Aussi, ces compagnies aériennes sont plus sensibles aux demandes des îles qui font des efforts de promotion considérables ${ }^{(4)}$ et qui investissent pour développer leurs produits touristiques (casinos, parcs, politiques de commerce hors-taxe avantageuses, etc.). Le déplacement d'une escale d'une île à l'autre dans le trajet d'un vol intercontinental a des implications immédiates sur la distribution des flux touristiques. Ces escales, comme celles des croisières, sont courtes mais vitales. Les îles font aussi des pressions pour obtenir des réductions des tarifs aériens bien que cela puisse comporter des dangers. Les baisses de prix du début des années 80 ont sûrement fait augmenter la quantité d'européens et de nord-américains arrivant dans le Pacifique Sud mais les australiens ont également profité des bas prix pour voyager outre-mer.

Les îles du Pacifique à forte vocation touristique pourraient tirer profit d'une éventuelle concertation afin de maximiser les recettes potentielles du tourisme international( ${ }^{(5)}$. Par le passé, leurs produits hétérogènes - mais facilement substituables - les ont poussées à prendre des décisions indépendantes (a agir comme en concurrence monopolistique). Les industries touristiques nationales réduisaient leurs prix et les bênéfices dérivés du tourisme ne dépassaient guère les coûts qui y étaient associés. Si les intếressés se concertaient, il serait possible d'augmenter les prix des services touristiques (transport international exclu) afin de maximiser les recettes totales pour la région et de planifier la distribution des services de transport aériens. Ces actions serviraient sûrement plus l'intérêt des pays d'accueil que celui des touristes (ils agiraient comme des oligopoles différenciés). Évidemment, il existe d'importantes rúticences pour ces pays qui n'ont acquis leur independance politique que récemment. Des hausses de prix contrồlées de l'hébergement et des autres services consommés par les touristes internationaux ne devraient pas menacer la position concurrentielle de la région dans son ensemble car les flux de touristes sont surtout affectés par le cout du transport aérien. Les autres services touristiques demeureraient passablement moins chers que ceux d'Hawail par exemple.
Les pays en voie de développement de I'Asie du Sud-Est

Au mẻme titre que les îles du Pacifique, les pays en voie de développement de l'Asie du Sud-Est projètent des images paradisiaques dans l'esprit des consommateurs d'exotisme. Les pays de cette région qui sont officiellement ouverts au tourisme international sont regroupes à des fins de cooperation economique intra-régionale au sein de I'A.S.E.A.N. (Association of South East Asian Nations). Il s'agit de l'Indonésie, de la Malaisie, des Philippines, de la Thaillande et de Singapour. Ces pays sont différents des Îles du Pacifique par leurs dimensions, leurs importantes populations et le degre de diversification de leurs économies. Ils ont cependant des problèmes très similaires au niveau du besoin de devises et de l'urgence à hausser le niveau de vie de leurs habitants. Plusieurs auteurs se sont intéressés à l'impact de la dimension des projets touristiques sur les profits économiques, sociaux et culturels bouleversant ces sociétés. D'autres se sont penchés sur l'association croissante du phénomène touristique avec celui de la prostitution $^{(6)}$. Ce lien fait que dans certains pays (''Australie, par exemple), le voyage en Thailande ou aux Philippines d'un homme seul est presqu'automatiquement associé à la recherche de plaisirs sexuels dans l'esprit de son entourage. Pour traiter de la promotion du tourisme international, il est utile de se pencher sur deux cas bien documentés, Bali, (Indonésie) et les Philippines.

Le décor et la richesse culturelle de Bali ont rapidement promu cette île comme destination privilégiée pour le tourisme de masse. Bien que les Australiens constituent son marché principal, la tendance est à la diversification des lieux d'origines des touristes et à une augmentation de la présence asiatique. La stratégie du gouvernement face au flot croissant des touristes füt de développer un complexe touristique de type "enclave" et un aéroport international afin de concentrer les services nécessaires. Depuis, le débat est très vif sur les coûts et bénéfices de cette approche $^{(7)}$. Ceci a eu un impact majeur sur les efforts de promotion des investisseurs et du gouvernement et explique leurs hésitations quant aux stratégies à suivre. De façon cruciale, ces efforts dépendent du type de développement qui sera finalement choisi.

Aux Philippines, les infrastructures ont été concentrées dans les grandes villes et surtout autour de Manille. La mise en place fort lente de l'administration nationale du tourisme s'est surtout centrée sur la promotion des activités de congrès. Ceci a eu pour résultats de favoriser des sejjours de touristes internationaux assez courts et concentrés dans les villes principales; les bénéfices du tourisme atteignent rarement les régions périphériques ${ }^{(i)}$. Le gouvernement philippin a développé aussi des politiques agressives en matière de transport aérien qui ont apporté des changements remarquables dans les flux. de touristes. Les arrivées intra-régionales et en provenance du Japon ont crû suffisamment pour créer des malaises au sein de l'industrie. Les Philippines, ainsi que d'autres pays de l'A.S.E.A.N., se plaignent du fait que les japonais ont l'habitude de voyager sur leurs propres compagnies aériennes, de demeurer dans des hôtels appartenant à des japonais, de manger dans des restaurants japonais et même de faire planifier leurs activités et visites locales par les agenoes de voyages japonaises. Conséquemment, l'effort de promotion des autorités phillipinoises prendra probablement une nouvelle direction dans un proche futur et cherchera a attirer une part plus grande de touristes d'outre-mer.

\section{Les "nouveaux" pays industrialisés}

Cette catégorie est composée de nations qui donnent des images de leurs produits touristiques complètement differentes des cas précédents. La Corée du Sud, Hong Kong, Taïwan et mẻme Singapour sont cependant des pays très hétérogènes, tant au niveau de leurs cultures et de leurs contextes ́́conomiques qu'au niveau de leurs produits touristiques. Ils ont cependant en commun leur dynamisme industriel et leurs positions comme plaques tournantes du commerce asiatique.

Hong Kong présente un intérêt particulier à cause de son importance comme base de correspondance aérienne, de sa position géographique et surtout de sa situation comme "centre" commercial. Le "shopping" est le principal atout de ce territoire qui n'a reconnu le tourisme comme activité économique majeure qu'au début des annés 80 . L'Association Touristique de Hong Kong (HKTA), financée par la taxation de l'industrie, a pour objectif majeur de stimuler la demande sans intervenir ou réglementer; ceci est typique de l'esprit de ce pays ${ }^{(9)}$. La promotion s'est effectuée en priorité auprès des segments de marché qui dépense beaucoup; il fallait essayer de compenser la capacite restreinte de l'industrie et l'inflation qu'elle a entrainée au début des années 80 . On espère accroître les revenus de l'industrie en se concentrant sur les voyages thématiques et sur les conventions mais des touristes indépendants arrivent a Hong Kong, chaque année, de plus de cent pays différents. Les résultats de l'effort promotionnel ont éttế jusqu'à prếsent peu concluants. La demande, semble-til, répond davantage aux variations de la situation économique internationale qu'aux efforts de promotion.

Des problèmes structurels reliss à l'offre touristique affectent aussi la croissance des arrivées de touristes. Les taux d'occupation des hôtels sont tels que les grossistes et congressistes ont peine a faire des réservations pour des groupes imposants et une augmentation de la capacité d'hébergement est difficilement envisageable; de plus il y a un manque de main d'oeuvre qualifiée en tourisme. L'aéroport lui-même, en perpétuelle amélioration, ne parvient pas à satisfaire les besoins 
du marché. Par contre, l'ouverture de la Chine aux touristes apparaît comme une tendance opportune pour Hong Kong qui fait presque toujours partie des circuits de cette région.

Aussi, l'image de Hong Kong se dégrade lentement. Le mythe de la coexistence de la société moderne et de la culture orientale est menace par les bonds de sa modernisation. Le gouvernement se voit maintenant obligé de subventionner un festival culturel afin de redorer son blason. Même les produits artisanaux sont importés de Chine en plus grand nombre et revendus plus chers à Hong Kong.

\section{Les économies post-industrielles}

Le Japon, l'Australie et la Nouvelle-Zélande constituent des sociétés économiquement mieux portantes que leurs voisines du Pacifique. Récemment, l'Australie a fait des efforts très considérables pour attirer le tourisme international. Ses marchés historiquement importants ont décliné (principalement la Grande-Bretagne) et son marché principal s'est fait plus volatile (les néo-zélandais comptent pour environ un tiers des arrivées en Australie); il a fallu essayer de diversifier les origines des touristes internationaux pour éviter toute dépendance envers certaines liaisons aériennes stratégiques et pour réduire la saisonnalité des arrivées (les tou-

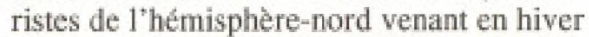
et les néo-zélandais, l'été). Une des stratégies mises de l'avant entre 1972 et 1980 fût la diversification des services aériens en termes de qualité, de conditions de durée de séjour et du nombre d'escales pour les vols outre-mer ${ }^{(10)}$. Les tarifs aériens ont toujours été la principale barrière du tourisme en Australie et on a essayé de segmenter le marché. afin d'attirer d'autres types de voyageurs que ceux visitant parents et amis.

Cependant, Qantas, la compagnie aérienne nationale, les autres transporteurs, les États et le gouvernement central agissaient de façon indépendante sans aucun effort de coordination pour structurer la promotion de l'Australie. Le résultat fut une distribution régionale assez inégale des bénéfices du tourisme international (concentré à l'est du pays) et surtout une performance assez faible. Sans expliquer cette faiblesse concurrentielle de 1'Australie par rapport aux autres destinations d'Asie et du Pacifique, son isolement ne suffisait pas: on a accuse son image sans relief ${ }^{(1)}$. Il a donc fallu definir un visage particulier du tourisme australien plus riche que celui des plages et des kangourous. Le contraste entre Sydney, moderne et vivante, et le "bush", la prairie désertique australienne peuplée d'aborigènes et d'une faune unique sont des élếments qu'on veut maintenant exploiter.

Les actions les plus récentes du gouvernement sont notamment une intense campagne de promotion aux Etats-Unis, entreprise explicitement pour "trapprocher" I'Australie en la démystifiant ("same color, same lan- gage" "). On veut présenter le pays comme la "dernière frontière" mais en rappelant qu' on y parle anglais et qu'on y est "blancs"(12) Au cours des cinq derniêres années, l'Australie s'est efforcée de diverses façons d'affirmer sa place sur la carte du tourisme international:

1) Une meilleure distribution des dépliants sur les prestations touristiques australiennes dans les agences de voyages nordaméricaines.

2) Des négociations bilatérales pour faire une promotion conjointe avec les transporteurs aériens étrangers présents sur les routes en expansions; du Japon, de l'Asie du Sud-Est et de l'Afrique du Sud. (Cette dernière destination est présentée comme une solution potentielle au problème des disparités régionales car les vols s'effectuent à partir de l'Australie de l'Ouest).

3) Des services aéroportuaires de meilleure qualité dans presque toutes les villes australiennes.

4) Une adaptation des services aériens aux besoins particuliers de certains marchés Par exemple, la structure actuelle des tarifs avantage nettement les séjours de deux semaines alors que les japonais prennent souvent des vacances d'une semaine.

Présentement, l'Australie entière semble attendre "Mer et Monde" du tourisme international. Le gouvernement fait même une campagne intérieure, présente aux australiens la publicité faite aux États-Unis et demande à la population d'accueillir les touristes avec le sourire. Le bas taux de change, 1"*Americas Cup' 'a Perth, 1'Expo 88 à Brisbane, le succès américain de "Crocodile Dundee" et la victoire de Pat Cash à Wimbledon semblent donner un bon coup de main à l'effort promotionnel général; mais le mauvais goût de certains investissements (Surfer's Paradise au Queensland est une réplique de Fort Lauderdale) et l'incertitude face aux coûts et aux bénéfices réels ne plaisent pas à bien des observateurs. Le tourisme ne serait-il qu'une nouvelle ruée vers l'or en Australie?

\section{Perspectives générales}

Le tourisme international dans le Pacifique en est encore à un niveau élémentaire. Bien que les coins les plus reculés de la planète soient maintenant accessibles, seules quelques destinations "tprivilégiées"' reçoivent les flots du tourisme de masse. Les efforts de promotion des pays de la région tendent à s'inscrire de plus en plus à l'intérieur de stratégies globales ou les intérèts sociaux, économiques et culturels sont pris en considération. Les traits communs à toute la region sont la reconnaissance du transport aérien comme déterminant majeur de la demande et l'interdépendance des différents pays. Ces destinations nè sont souvent que des parties de circuits touristiques et ne constituent généralement qu'une petite fraction d'un produit global. Les pays les plus dynamiques essaient de différencier leurs produits touristiques de celui de leurs voisins en se créant des images propres. La diversification des marchés semble aussi être une préoccupation commune afin d'étaler le tourisme sur toutes les saisons et d'éviter toute dépendance géo-politique.

L'avenir du tourisme dans la région est somme toute assez prometteur, la Chine créant un nouveau póle d'attraction, les japonais voulant dépenser et trouver de nouveaux marchés commerciaux et les australiens étant pris d'une "frénésie pro-tourisme". La croissance des arrivées internationales devrait done se maintenir assez ellevée. Les seuls obstacles possibles présentement seraient les renversements politiques peu prévisibles. Après les événements des Philippines et de la Nouvelle-Calédonie, Fiji a vu ses arrivées de touristes diminuer de $70 \%$ (selon les média australiens?). Comment ces faits géo-politiques peuvent-ils affecter le tourisme de la région dans son ensemble? Voilà une nouvelle interrogation pour la région du Pacifique $f$

\section{Notes et références:}

(1) Q.M.T.. Etude économique du tourisme mondial. 1984, P. 41 .

2) VELLAS, Francois, Économis et politique du tourisme intemational, Economica, Paris, 1966.

(3) BRITTON, Stephen G., Tourism and underdevelopment in Fiji, Australian National University, Development Studies Centre, monograph no. 31. 1983.

(4) Idem.

(5) RAJOTTE, Dr. F., The impact of tourism development in the Pacific, University of the South Pacific, 1962.

(6) COHEN, Eric, Thar girls and Farang men: the edge of ambiguity. Annals of tourism research, vol. 9, no 3, 1982, pp. $403-428$.

(7) NORONHA, Raymond, Paradis retrouve: Tourisme a Ball, Tourisme - Passeport pour le doveloppement?, Economica, Paris, 1990, pp. 177-204.

(8) DELOS SANTOS, J.S, ORTIZ, E.M., HUANG, E., SECRETARIO. $F_{1}$, Philispines, Tourism in Asia: The Economic Impact, Singapore University Press, 1983, pp. 173-240.

(9) LIN, T.-B., SUNG, Y.-W., Hong Kong. Tourism in Asia: The Economic Impect, Singapore University Prass, 1983, pp. 1-100.

(10) Department of Industry and Commerce, Australiar Gowernment, Proceedings of the National Tourism Outlook Conference - 1981, Australian Government Publishing Services, Canberra, 1982.

(11) Idem.

(12) Idem. 